OPEN ACCESS

Edited by:

Xiaoxing Xiong,

Wuhan University, China

Reviewed by:

Jorge Tolivia,

University of Oviedo, Spain

Ping Gong,

Dalian Medical University, China

*Correspondence:

Yong-Mei Zhang

zhangym700@xzhmu.edu.cn

tThese authors have contributed equally to this work

Specialty section:

This article was submitted to

Multiple Sclerosis and

Neuroimmunology,

a section of the journal

Frontiers in Neurology

Received: 25 May 2019 Accepted: 26 November 2019 Published: 12 December 2019

Citation:

Ji N-N, Wu L, Shao B-M, Meng Q-X, $X u J-N$, Zhu H-W and Zhang Y-M

(2019) CTL-Derived Granzyme $B$ Participates in Hippocampal Neuronal Apoptosis Induced by Cardiac Arrest and Resuscitation in Rats.

Front. Neurol. 10:1306

doi: 10.3389/fneur.2019.01306

\section{CTL-Derived Granzyme B Participates in Hippocampal Neuronal Apoptosis Induced by Cardiac Arrest and Resuscitation in Rats}

\author{
Ning-Ning $\mathrm{Ji}^{1+}$, Liang $\mathrm{Wu}^{1,2+}$, Bo-Ming Shao ${ }^{1 \dagger}$, Qing-Xiang Meng ${ }^{1}$, Jin-Nan $\mathrm{X} u^{3}$, \\ Hao-Wen Zhu ${ }^{3}$ and Yong-Mei Zhang ${ }^{1,3 *}$ \\ ${ }^{1}$ Jiangsu Province Key Laboratory of Anesthesiology, Xuzhou Medical University, Xuzhou, China, ${ }^{2}$ Anesthesiology \\ Department of the First People's Hospital of Xuzhou, Xuzhou, China, ${ }^{3}$ School of Anesthesiology, Xuzhou Medical University, \\ Xuzhou, China
}

Hippocampal neuronal apoptosis is a devastating consequence of cardiac arrest (CA) and subsequent cardiopulmonary resuscitation (CPR). In this study, we assessed the contribution of cytotoxic $T$ lymphocyte (CTL)-derived toxic mediator granzyme B (Gra-b) to the hippocampal neuronal apoptosis following CA/CPR in rats. Rats that experienced CA/CPA presented with cytosomal shrinkage, dense cytoplasm, and intensive eosinophilic staining in the CA1 region of dorsal hippocampus. CA/CPR rats also exhibited inability in spatial navigation and a local infiltration of peripheral CD8+ $T$ cells into the hippocampus. The protein levels of Gra-b, cleaved Caspase-3, and cleaved PARP1 were significantly elevated in rats undergoing CA/CPR. Pretreatment with Gra-b inhibitor suppressed Gra-b release, attenuated hippocampal neuronal apoptosis, as well as improved cognitive impairment. Together, this study indicates that CTL-derived Gra-b is involved in the CA/CPR-induced neuronal apoptosis, and pharmacological manipulation of Gra-b may represent a novel avenue for the treatment of brain injury following CA/CPR.

Keywords: cardiac arrest, cardiopulmonary resuscitation, hippocampus, cytotoxic T lymphocytes, granzyme B, apoptosis

\section{INTRODUCTION}

Cardiac arrest (CA), an abrupt loss of heart function in individuals with or without heart disease, is a leading cause of cardiovascular and cerebral hypoxia and ischemia, resulting in disability and mortality (1). Despite the application of cardiopulmonary resuscitation (CPR) to reduce the mortality from CA, brain ischemia remains a devastating complication of CA/CPR, with considerable neuronal apoptosis and cognitive deficits within the initial 3 months after CPR in $\sim 70 \%$ of the survivors $(2,3)$. To date, there is a paucity of approaches with clinical efficacy, reliability, and validity to prevent brain injury following CA/CPR (4).

Sustained CA and hypoxia induced ischemic brain injury with the onset of secondary neuroinflammation, including glial activation, peripheral immune cell recruitment, and pro-/antiinflammatory factor release, resulting in pyrexia, hypophagia, hyperalgesia, and cognitive 
dysfunction (5). Meanwhile, the inflammatory responses facilitate cell fragment clearance, tissue repair, and functional recovery (6). In addition, studies of the central nervous system (CNS) evidenced an elevation of CD8+ cytotoxic T lymphocytes (CTLs) in a number of pathogen-induced neurological disorders (7-9), wherein activated CTLs can generate a series of serine proteases, particularly granzymes (Gra), which can induce cellular apoptosis, leading to neuronal death in ischemic brain regions (10-12). Moreover, Gra-b reportedly participates in the development of neurological diseases, such as brain ischemiareperfusion injury (13). Despite the de facto infiltration of CTLs into the CNS after CA/CPR, the precise contributions of CTL-derived Gra-b to neuronal apotosis remain elusive. Herein, we aimed to investigate the effects of CTLs-derived Gra-b on the modulation of hippocampal neuronal apoptosis in a rat model of CA.

\section{MATERIALS AND METHODS}

\section{Animals}

Adult male Wistar rats (weighing 250-300 g) were purchased from Jining Lukang Animal Co. Ltd. (Shandong, China) and housed in a $12 \mathrm{~h}$ light and dark cycle (lights on at 7:00 a.m.) with ad libitum access to food and water. All experimental protocols were approved by the Institutional Animal Care and Use Committee of Xuzhou Medical University [SYXK (Su) 2010-2011].

\section{Experimental Procedures}

Rats were mainly randomized into sham $(n=15), \mathrm{CA} / \mathrm{CPR}(n=$ $20)$, and Gra-b inhibitor $(n=20)$ groups according to random number table. The CA/CPR model was established by asphyxiainduced CA and subsequent CPR as described previously (14). In brief, the ventilator connected with trachea was disconnected to induce hypoxic CA in anesthetized rats. CPR was implemented by manual precordial compressions and mechanical ventilation after $6 \mathrm{~min}$ of untreated cardiac arrest. Manual precordial compressions were maintained at a rate of about 200 per minute. Compression depth was $\sim 30 \%$ of anteroposterior chest diameter at maximal compression. Ventilation was resumed by a volume controlled small animal ventilator with a frequency of 100 breaths-per-minute, an inspired $\mathrm{O}_{2}$ fraction of 1.0 and a tidal volume of $6 \mathrm{ml} / \mathrm{kg}$. Ventricular fibrillation, if appropriate, was removed with up to three 2 -J shocks after $8 \mathrm{~min}$ of CPR. If restoration of spontaneous circulation (ROSC) was not achieved, a $30 \mathrm{~s}$ interval of CPR was performed before a subsequent sequence of up to 3 shocks was attempted. This procedure was repeated for a maximum of three cycles. ROSC was defined as a return of supraventricular rhythm with a mean aortic pressure above $50 \mathrm{mmHg}$ for a minimum of $5 \mathrm{~min}$. In the case of spontaneous respiration, the ventilator was powered off. Grab inhibitor I $(0.5 \mathrm{mM} / \mathrm{kg}$ body weight; 368050, Calbiochem, USA) was administered immediately after ROSC via a femoral catheter. Sham group only received identical surgical procedures except asphyxia.

\section{Cerebral Performance Category (CPC) and Morris Water-Maze (MWM)}

The neurological deficiency score after CA/CPR was recorded for 4 consecutive days according to CPC scoring system in which scores range from 0 to 5 based on consciousness, motor function, and sensory function. 0 stands for normal status, 1 for mild cerebral disability, 2 for moderate cerebral disability, 3 for severe cerebral disability, 4 for coma/vegetative state, and 5 indicates brain death. Morris water-maze testing was conducted as described previously (15).

\section{Blood-Brain Barrier Permeability}

Blood-brain barrier (BBB) permeability was detected by measurement of the Evans blue (EB) extravasation (16). EB dye ( $4 \%$ in $0.9 \%$ saline) was injected into the caudal vein $(4 \mathrm{~mL} / \mathrm{kg})$. Two hours afterwards, rats were transcardially perfused prior to the isolation of ischemic hemisphere. The EB level in brain tissue was determined by spectrophotometry at a wavelength of $660 \mathrm{~nm}$.

\section{Hematoxylin and Eosin Staining}

Three days after $\mathrm{CA} / \mathrm{CPR}$, rats were transcardially perfused with $0.9 \%$ saline followed by $4 \%$ paraformaldehyde under deep anesthesia. Rat brains were isolated and post-fixed with paraformaldehyde for another $24 \mathrm{~h}$ prior to embedment in paraffin. The rat hippocampus was coronally sliced at $4 \mu \mathrm{m}$ and stained with hematoxylin and eosin.

\section{Western Blotting}

Rat hippocampus was homogenized in RIPA lysis buffer, with $50 \mu \mathrm{g}$ protein sampled for SDS-PAGE. After the protein transference, the PVDF membrane was rinsed in washing buffer for $5 \mathrm{~min}$, followed by addition of $5 \%$ skim milk powder, at room temperature $(\mathrm{r} / \mathrm{t})$ for $2 \mathrm{~h}$. Blots were then incubated in anti- $\beta$-actin (1:1,000, rabbit, Sigma-Aldrich), anti-Gra-b (1:200, rabbit, Abcam), anti-caspase 3 (1:200, rabbit, Santa Cruz), or anti-PARP1 (1:200, rabbit, Santa Cruz) overnight at $4^{\circ} \mathrm{C}$. On the following day, the PVDF membranes were maintained at $\mathrm{r} / \mathrm{t}$ for $30 \mathrm{~min}$, and were thereafter rinsed with the washing buffer for $10 \mathrm{~min}$ in triplicate, followed by incubation with anti-rabbit IgG with alkaline phosphatase (1:1,000, A0208, Beyotime, China) on the shaking table at $\mathrm{r} / \mathrm{t}$ for $2 \mathrm{~h}$. The blots were developed by nitro-blue tetrozolium/bromochbating in alkaline phosphate (NBT/BCIP) substrate after incubation in alkaline phosphataseconjugated secondary antibodies for $2 \mathrm{~h}$ at $\mathrm{r} / \mathrm{t}$. ImageJ software was employed for grayscale analysis.

\section{Immunofluorescence Analyses}

The hippocampus was sliced at $30 \mu \mathrm{m}$ thickness with a cryostat (Leica CM1800; Heidelberg, Germany). For immunofluorescence staining, slices were blocked by $10 \%$ goat serum for $120 \mathrm{~min}$ and incubated with primary antibodies, including anti-CD-8 (1:100, mouse, Abcam), anti-TCR (1:100, mouse, Abcam), anti-Gra-b (1:100, rabbit, Abcam), anti-NeuN antibody (1:50, rabbit, CST; 1:50, mouse, Millipore), anti-Annexin V antibody (1:100, goat, Proteintech) for $24 \mathrm{~h}$ at $4^{\circ} \mathrm{C}$ following by incubation in secondary antibodies for $1 \mathrm{~h}$ at $37^{\circ} \mathrm{C}$ and mounted with $90 \%$ glycerol. The 
sections were visualized by a Leica confocal microscope. ImageJ Pro software was adopted for cell quantification.

\section{Statistical Analysis}

All data are expressed as mean \pm SEM. CPC scores and MWM acquisition data were analyzed by two-way repeated measures analysis of variance (ANOVA) followed by Tukey's post-hoc multiple comparisons. The number of CD8+ cells and apoptosis cells were analyzed by unpaired Student's $t$-test. The data of WB assays were analyzed by one-way ANOVA followed by post-hoc Student's Newman-Keuls (SNK) test. $p<0.05$ was considered statistically significant.

\section{RESULTS}

There was no significant difference in the survival rate at $24 \mathrm{~h}$ after resuscitation between the CA/CPR and inhibitor groups $(12 / 20$ vs. $12 / 20 ; P>0.05)$. Then half of the rats resuscitated successfully from cardiac arrest were used for immunofluorescence staining and HE staining $(n=6)$, and the rest of the rats were used for western blotting $(n=6)$.

\section{CA/CPR-Induced Hippocampal Neuronal Apoptosis}

The interval between asphyxia and arrest was usually 5-6 min. Spontaneous circulation (ROSC) was restored about 1 min after $\mathrm{CPR}$. The rats were subjected to $\mathrm{CA} / \mathrm{CPR}$, and were euthanized $24 \mathrm{~h}$ thereafter, with the CA1 region of dorsal hippocampus isolated and assessed by $\mathrm{H}-\mathrm{E}$ staining and immunofluorescence labeling of Annexin V (Figure 1A), respectively. The H-E staining indicated cytosomal shrinkage, dense cytoplasm, and intensive eosinophilic staining of the neurons, indicating neuronal apoptosis. In parallel, the immunofluorescence assay revealed the expression of hippocampal Annexin V, a protein marker of early-stage apoptosis, was significantly increased as compared to that in the sham group (Figure 1C). The Annexin V-positive cells in the CA1 pyramidal cell layer in the dorsal hippocampus from CA/CPR rats significantly multiplied as compared to the sham rats $(24.67 \pm 0.88$ vs. $7 \pm 1.53$; $t_{(4)}=-10.02, p=0.001$; Figure 1B).

\section{Treatment With Gra-b Inhibitor Alleviated CA/CPR-Induced Behavioral Impairment}

The CA/CPR rats displayed shuffling gait, decline in consumption of water and food, as well as neurologic and cognitive deficits. Administration of Gra-b inhibitor abated the cerebral performance category (CPC) scores in CA/CPR rats. A two-way repeated measures ANOVA revealed significant main effects in duration $(p<0.001)$, manipulation mode $(p<0.001)$, and significant duration $\times$ manipulation interaction $(p<0.001)$. Post-hoc Bonferroni multiple comparisons showed that elevated CPC scores in CA/CPR rats (CPR vs. Sham, $p<0.001)$ and Gra-b inhibitor annulled the elevation of CPC scores (CPR vs. inhibitor, $p<0.001$; Figure 2A).

Gra-b inhibitor improved the spatial navigation performance in a Morris water maze (MWM) task in CA/CPR rats. A two-way repeated measures ANOVA revealed significant main effects in duration $(p=0.000)$, manipulation $(p<0.001)$, and significant duration $\times$ manipulation interaction $(p<0.001)$. Post-hoc Bonferroni multiple comparisons showed that CA/CPA impaired the spatial learning ability (CA/CPR vs. Sham, $p<$ 0.001 ) and Gra-b inhibitor alleviated the impairment in spatial navigation (CA/CPR vs. inhibitor, $p=0.01$; Figure 2B).

Gra-b inhibitor improved the retrieval of spatial memory in an MWM task in CA/CPR rats. A one-way ANOVA indicated a significant difference ( $p=0.014$; Figure 2C). Post-hoc Bonferroni multiple comparisons showed reduced duration in target zone for $\mathrm{CA} / \mathrm{CPR}$ rats $(p=0.012)$, which was reversed by Gra-b inhibitor.

The blood-brain barrier (BBB), in integrity, plays a protective role to the organism, whereas its disintegration leads to the infiltration of peripheral immune cells via the site of lesion. Hence, the permeability of BBB following CA/CPR was evaluated by Evans Blue (EB) assay $24 \mathrm{~h}$ after CA/CPR. The EB concentration was significantly higher in the CA/CPR group than in the sham group, which demonstrated that CA/CPR increased BBB permeability ( $p<0.001$, Figure 2D). Post-hoc Bonferroni multiple comparisons showed an increase in EB level at 12 and $24 \mathrm{~h}$ after CA/CPR (all $p<0.01$ ). Thereafter, a multitude of CD8 T cells were identified to have infiltrated into hippocampus. In parallel, Western blotting assay showed upregulated expression of the CD8 protein in CPR group $(p<$ 0.001). Post-hoc Bonferroni multiple comparisons showed the increased CD8 protein at 6, 12, and $24 \mathrm{~h}$ after CPR (all $p<0.05$; Figure 2E). Additionally, the CD8 signal was predominantly co-localized with the $\mathrm{T}$ cell marker $\mathrm{T}$ cell receptor (TCR) (88\%) and CD8/TCR-positive cells accounted for 36\%, based on immunofluorescence staining (Figures 2F,G).

\section{CA/CPR-Induced Elevation of Hippocampal Gra-b Level}

The expression of hippocampal Gra-b was assessed to explore the role of $\mathrm{CD} 8+\mathrm{CTL}$ in $\mathrm{CA} / \mathrm{CPR}$-induced brain injury. Immunofluorescence staining revealed that TCR signal was dominantly co-localized with the Gra-b protein, suggesting that $\mathrm{CD} 8+\mathrm{CTL}$ serving as the origin of Gra-b protein (Figure 3A). CA/CPR rats exhibited an increase in Gra-b protein expression and co-localization with NeuN (Figure 3B). CA/CPR upregulated the Gra-b protein expression as demonstrated by a one-way ANOVA $(p=0.003)$. Post-hoc Bonferroni multiple comparisons revealed the increased Gra-b level after CPR (all $p<0.05$; Figure 3B).

CA/CPR upregulated the cleaved Caspase-3 protein expression as validated by a one-way ANOVA $(p<0.001$; Figure 3C). Post-hoc Bonferroni multiple comparisons showed the increased level of cleaved Caspase- 3 as from 3 to $72 \mathrm{~h}$ after CPR (all $p<0.01$; Figure 3C). CA/CPR upregulated the cleaved PARP1 protein expression as demonstrated by a one-way ANOVA ( $p=0.042$; Figure 3C). Post-hoc Bonferroni multiple comparisons showed the increased level of cleaved PARP1 protein after CPR (all $p<0.05$; Figure 3C).

Gra-b inhibitor is well-documented to modulate apoptosis, which would incite interest to ascertain whether and how the apoptosis-signaling pathway in the rat brain was involved in this 
A

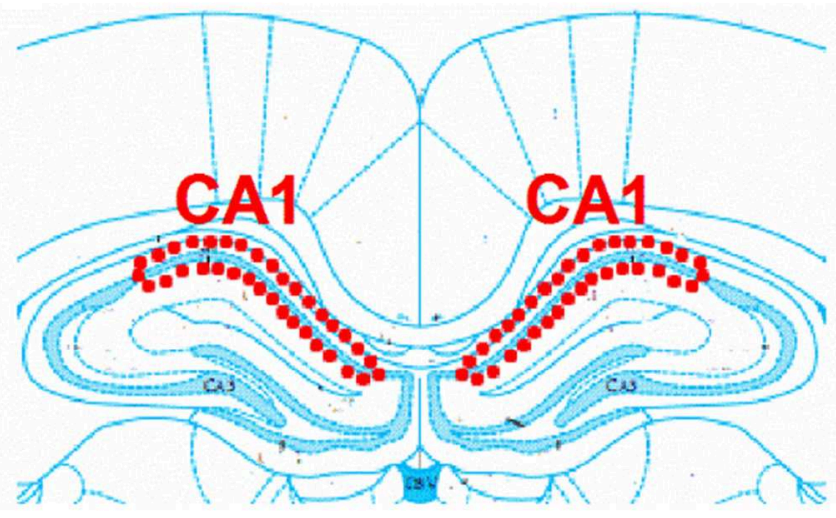

C HE
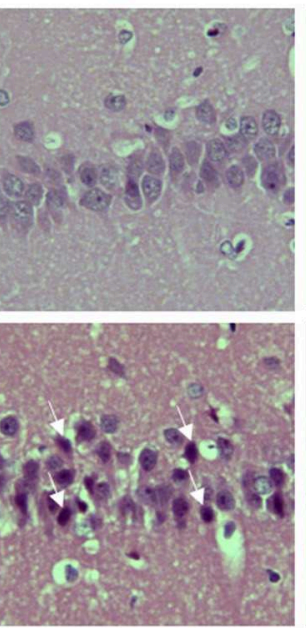

Annexin-V
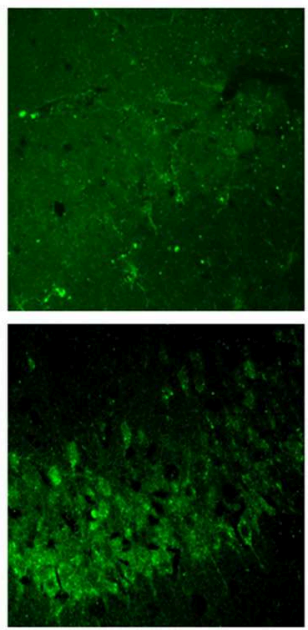

Sham

CA/CPR
B

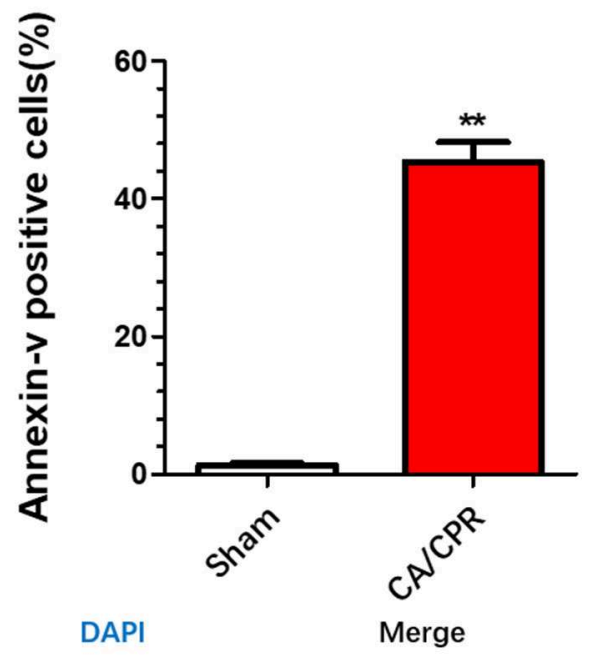

FIGURE 1 | CA/CPR induced hippocampal neuronal apoptosis. The immunofluorescent staining was conducted in the CA1 region of dorsal hippocampus $24 \mathrm{~h}$ after CAVCPR. (A) Diagram of hippocampus. (B) The percentage of Annexin $\mathrm{V}^{+}$cells in total $\mathrm{NeuN}^{-}$cells was calculated. CAVCPR rats presented with a significant increase in the Annexin $\mathrm{V}^{+}$cells as compared to the sham rats. (C) The $\mathrm{H}$-E staining showed that cytosomal shrinkage, dense cytoplasm, and intensive eosinophilic staining of the neurons, and the immunofluorescence assay revealed the expression of hippocampal Annexin V, a protein marker of early-stage apoptosis, was significantly increased as compared to that in the sham group. The hippocampal neurons were labeled with NeuN, a neuronal marker. Scale bar $=20 \mu \mathrm{m}$. Data are expressed as mean \pm SEM; $n=6 ;{ }^{* *} p<0.01$.

process. Western blotting was employed to detect the variations of protein levels of Gra-b, cleaved Caspase-3, and cleaved PARP1. One-way ANOVA revealed that Gra-b inhibitor significantly reversed the upregulation of Gra-b $(p<0.001)$, cleaved Caspase- 3 $(p<0.001)$, and cleaved PARP1 $(p<0.001)$, respectively (Figure 3D).

\section{DISCUSSION}

Our present study confirmed that CA/CPR could increase the permeability of $\mathrm{BBB}$, allowing blood CD8+ CTLs to infiltrate into the brain tissue and secrete Gra-b protein, and leading to neuronal apoptosis and death, probably via the Caspase-3 signaling pathway. Administration of Gra-b inhibitor annulled the upregulation of Gra-b, cleaved Caspase-3, and cleaved PARP1 levels, thus ameliorating neuronal apoptosis as well as behavioral and cognitive impairment.

The brain was previously thought to be an immuneprivileged organ, containing resident immune cells that maintain the homeostasis of central nervous system. Recent studies demonstrated that the CNS has a canonical functional lymphatic system or meningeal lymphatic vessels, which directly connect deep cervical lymph nodes and promote the entry of immune cells into the brain $(17,18)$. Notwithstanding BBB prevents the immune cells from entry into the CNS, ischemic injury-induced $\mathrm{BBB}$ leakage allows peripheral immune cells to infiltrate into the brain parenchyma, and activate the resident microglia and astrocytes, or induce neuronal death via the direct action on the 


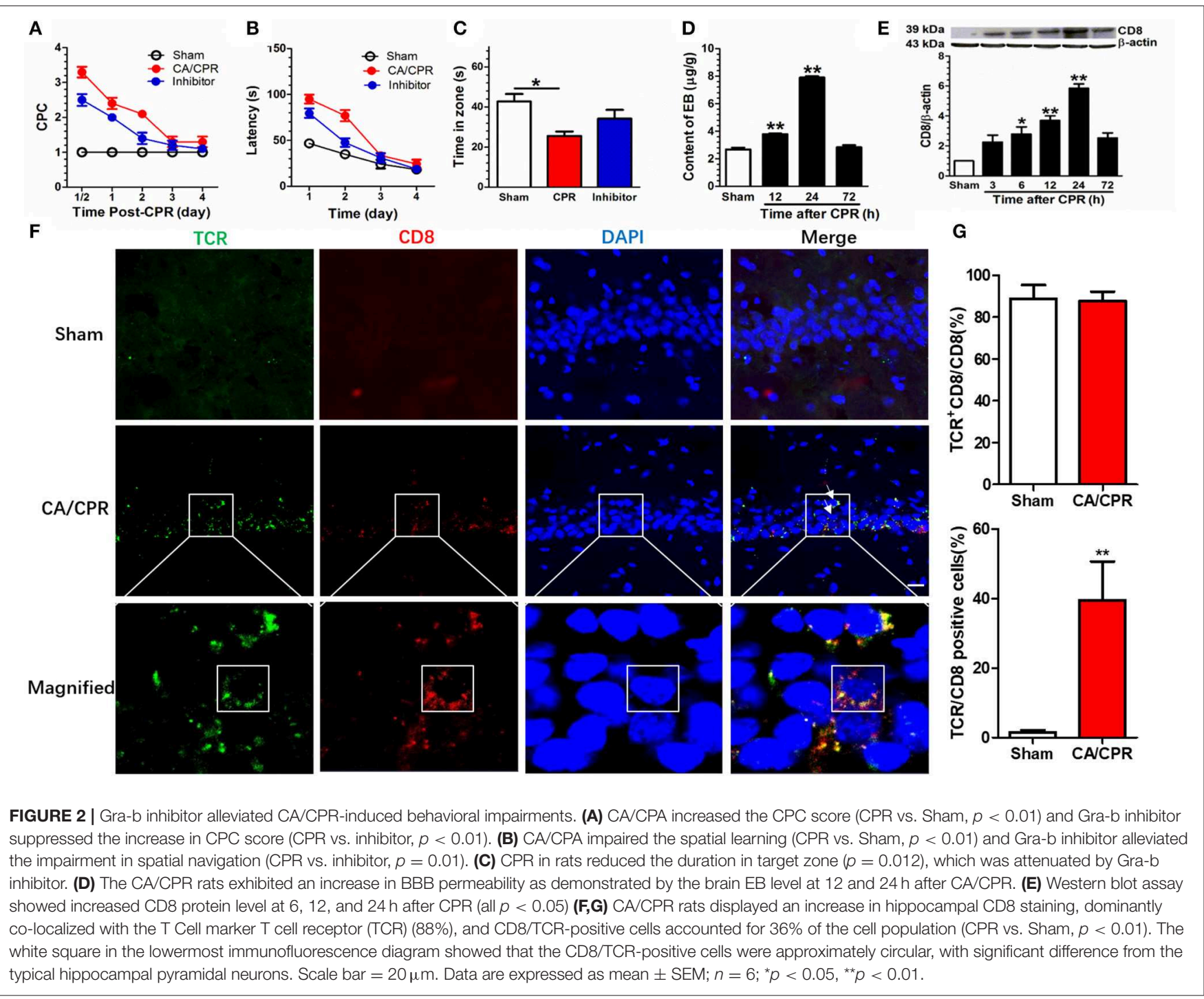

nervous system, as illustrated by the ability of CD8 $+\mathrm{T}$ cells to detect and destroy the MHC I-positive neurons (16).

The entry of blood $\mathrm{T}$ cells into the CNS may induce a delayed injury $(19,20)$. In the present study, CA/CPR could induce the infiltration and activation of CD8+ CTLs, with a scant population of glial cells existent in the hippocampus after CA/CPR (Supplementary Material). The peak levels of CD8+ CTLs in the hippocampus occurred at $24 \mathrm{~h}$ after $\mathrm{CA} / \mathrm{CPR}$. CA/CPR can induce brain ischemia and related adverse responses, e.g., $\mathrm{Ca}^{2+}$ overload, mitochondrial dysfunction, ATP depletion, pro-apoptotic protein expression, and glutamate excitotoxicity (21-23). Furthermore, CA/CPR can provoke inflammation, initiate immune responses, and induce cell death, which contributes to neurologic deficit and vulnerability of CA1 pyramidal neurons of the hippocampus to ischemic brain injury $(16,24)$.

The CD8 + CTLs can reportedly destroy target cells by the secretion of Gra-b or by contact. CD8+ knockout CTLs have been shown to decrease the infarcted area. Gra-b, a member of serine protease family, is a potent inducer of caspasedependent and independent forms of apoptosis. Once released by $\mathrm{T}$ cells and NK cells, Gra-b can directly cleave several substrates or pro-apoptotic proteins, e.g., PARP, to induce cell death (25). In addition, Gra-b is also capable of cleaving Bid into its truncated form (tBid), which subsequently migrates to the outer mitochondrial membrane to interact with $\mathrm{Bcl}-2$, and induce the release of Bad and Bax, with initiation of the intrinsic cell death pathway. Gra-b preferentially cleaves procaspases to initiate cell death in rats (26-30). PARP is a DNArepair enzyme, cleaved at aspartame residues to 89 and 21 $\mathrm{kDa}$ signature fragments by caspase during apoptotic cell death. Gra-b is involved in the neuronal degeneration in stroke in humans and neuronal death in cerebral ischemia in rats (13). In addition, inhibition of Gra-b could attenuate CD8 $\mathrm{T}$ cellinduced neurotoxicity and neuronal death. The present study revealed that the levels of Gra-b, cleaved caspase-3, and cleaved 


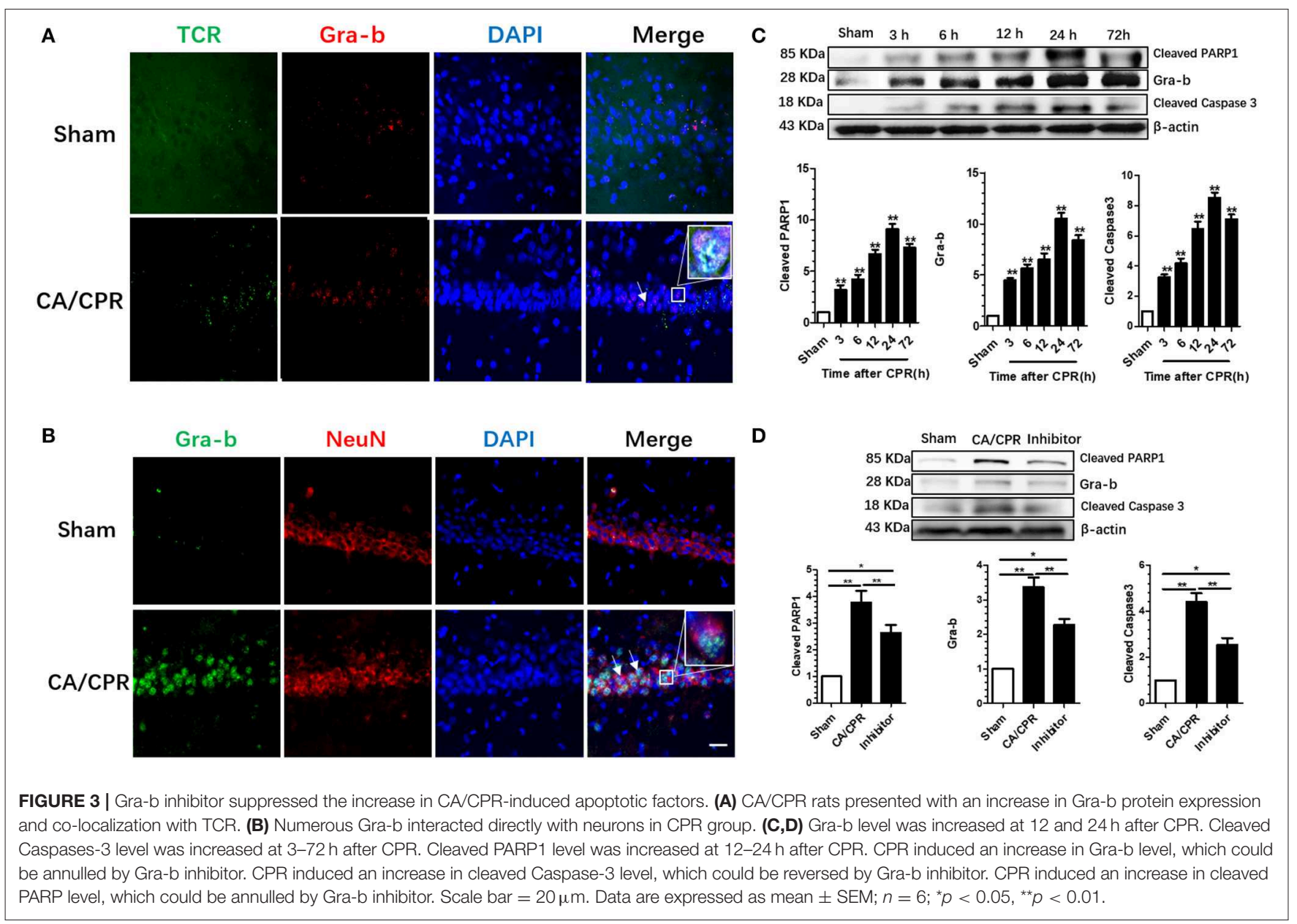

PARP1 increased at $3 \mathrm{~h}$ and reached the peak levels at $24 \mathrm{~h}$. Inhibition of Gra-b expression resulted in the downregulation of the expression of Gra-b, cleaved caspase-3, and cleaved PARP1, with the apoptosis of hippocampal CA1 neurons mitigated and neurological function improved. Hence, our results indicated that infiltration of $\mathrm{T}$ cells into the brain induced the secretion of Gra-b, contributing to neuronal apoptosis and ultimately, neuronal destruction.

Our study has limitations. The duration of brain injury after heart failure is a long process, whereas this study only focused on changes within the initial $72 \mathrm{~h}$ after CA/CPR. Animal experiments can only simulate instead of being equivalent to clinical scenario, and further studies which could extend the observation duration to verify the role of CTL-derived Gra-b in the process and prognosis of brain injury.

In summary, our current study evaluated whether CTL-derived Gra-b participated in neuronal apoptosis and death after CA/CPR. It is plausible that Gra-b induced neuronal apoptosis and death by activating caspase-3 and cleaving PARP1. The Gra-b inhibitor could suppress neuronal apoptosis and death, protect neuronal integrity, and improve the outcome. These data indicate Gra-b may be a key contributor of neuronal apoptosis and functional impairment after CA/CPR. Thus, we might postulate that modulation of Grab expression could attenuate the neuronal damages and benefit prognosis of CA/CPR. For the paucity and inefficacy of regimens for secondary brain injury after CA/CPR, suppression of Gra-b activity by pharmacological strategy may pave a novel avenue to therapeutics for CA/CPR -induced brain injury.

\section{DATA AVAILABILITY STATEMENT}

All datasets generated for this study are included in the article/supplementary material.

\section{ETHICS STATEMENT}

The animal study was reviewed and approved by the Institutional Animal Care and Use Committee at Xuzhou Medical University.

\section{AUTHOR CONTRIBUTIONS}

N-NJ contributed to the experimentation, data collection, and manuscript composition. LW, B-MS, J-NX, and H-WZ 
helped experiment performance. Q-XM assisted in data collection. Y-MZ contributed to the conception and supervision of the research, data interpretation, manuscript drafting, and revision. All authors have read and approved the submission and publication of the final version of manuscript. The authors vouch for the accuracy and completeness of the experiment.

\section{REFERENCES}

1. McCoyd M, McKiernan T. Neurologic complications of cardiac arrest. Handb Clin Neurol. (2014) 119:25-39. doi: 10.1016/B978-0-7020-4086-3.00003-5

2. Thom T, Haase N, Rosamond W, Howard VJ, Rumsfeld J, Manolio $\mathrm{T}$, et al. Heart disease and stroke statistics - 2006 update: a report from the American Heart Association Statistics Committee and Stroke Statistics Subcommitte. Circulation. (2006) 113:e46-215. doi: 10.1161/CIRCULATIONAHA.105.171600

3. Andresen M, Gazmuri JT, Marín A, Regueira T, Rovegno, M. Therapeutic hypothermia for acute brain injuries. Scand J Trauma Resusc Emerg Med. (2015) 23:42. doi: 10.1186/s13049-015-0121-3

4. Green DM. Hypothermia for cardiac arrest. Curr Neurol Neurosci Rep. (2006) 6:518-24. doi: 10.1007/s11910-006-0055-2

5. Hein AM, O'Banion MK. Neuroinflammation and memory: the role of prostaglandins. Mol Neurobiol. (2009) 40:15-32. doi: 10.1007/s12035-009-8066-Z

6. Fabry Z, Raine CS, Hart MN. Nervous tissue as an immune compartment: the dialect of the immune response in the CNS. Immunol Today. (1994) 15:218-24. doi: 10.1016/0167-5699(94)90247-X

7. Wu L, Ji NN, Wang H, Hua JY, Sun GL, Chen PP, et al. Domino effect of IL-15 and CD8 $\mathrm{T}$ cell-mediated neuronal apoptosis in experimental traumatic brain injury. J Neurotrauma. (2018). doi: 10.1089/neu.2017.5607. [Epub ahead of print].

8. Hurn PD, Subramanian S, Parker SM, Afentoulis ME, Kaler LJ, Vandenbark AA, et al. T- and B-cell-deficient mice with experimental stroke have reduced lesion size and inflammation. J Cereb Blood Flow Metab. (2007) 27:1798-805. doi: $10.1038 /$ sj.jcbfm. 9600482

9. Chaitanya GV, Babu PP. Multiple apoptogenic proteins are involved in the nuclear translocation of Apoptosis Inducing Factor during transient focal cerebral ischemia in rat. Brain Res. (2008) 1246:178-90. doi: 10.1016/j.brainres.2008.09.075

10. Kim JY, Ho H, Kim N, Liu J, Tu CL, Yenari MA, et al. Calcium-sensing receptor (CaSR) as a novel target for ischemic neuroprotection. Ann Clin Transl Neurol. (2014) 1:851-66. doi: 10.1002/acn3.118

11. Allen BS, Buckberg GD. Studies of isolated global brain ischaemia: I. Overview of irreversible brain injury and evolution of a new concept redefining the time of brain death. Eur J Cardiothorac Surg. (2012) 41:1132-7. doi: 10.1093/ejcts/ezr315

12. Voskoboinik I, Whisstock JC, Trapani JA. Perforin and granzymes: function, dysfunction and human pathology. Nat Rev Immunol. (2015) 15:388-400. doi: $10.1038 /$ nri3839

13. Chaitanya GV, Schwaninger M, Alexander JS, Babu PP. Granzyme-b is involved in mediating post-ischemic neuronal death during focal cerebral ischemia in rat model. Neuroscience. (2010) 165:1203-16. doi: 10.1016/j.neuroscience.2009.10.067

14. Gao CJ, Li JP, Wang W, Lü BC, Niu L, Zhu C, et al. Effects of intracerebroventricular application of the delta opioid receptor agonist [D-Ala2, D-Leu5] enkephalin on neurological recovery following asphyxial cardiac arrest in rats. Neuroscience. (2010) 168:531-42. doi: 10.1016/j.neuroscience.2010.02.025

15. Zhang G, Cinalli D, Stackman RW. Effect of a hallucinogenic serotonin 5-HT2A receptor agonist on visually-guided, hippocampal-dependent spatial cognition in C57BL/6J mice. Hippocampus. (2017) 27:558-69. doi: $10.1002 /$ hipo.22712

16. Owens, T. Identification of new therapeutic targets for prevention of CNS inflammation. Expert Opin Ther Targets. (2002) 6:203-15. doi: $10.1517 / 14728222.6 .2 .203$

\section{FUNDING}

This project was supported by grants from the National Natural Science Foundation of China (Nos. 81771203, 81772065), the Key Subject of Colleges and Universities Natural Science Foundation of Jiangsu Province (19KJA110001), and the Natural Science Foundation of Jiangsu Province (BK20161171).

17. Louveau A, Smirnov I, Keyes TJ, Eccles JD, Rouhani SJ, Peske JD, et al. Structural and functional features of central nervous system lymphatic vessels. Nature. (2015) 523:337-41. doi: 10.1038/nature14432

18. Russo MV, McGavern DB. Immune surveillance of the CNS following Infection and Injury. Trends Immunol. (2015) 36:637-50. doi: 10.1016/j.it.2015.08.002

19. Petito CK. Fred Plum Delayed hippocampal damage in humans following cardiorespiratory arrest. Neurology. (1987) 37:1281-6. doi: 10.1212/WNL.37.8.1281

20. Anthony DA, Andrews DM, Watt SV, Trapani JA, Smyth MJ. Functional dissection of the granzyme family: cell death and in?ammation. Immunol Rev. (2010) 235:73-92. doi: 10.1111/j.0105-2896.2010.00907.x

21. Lipton P. Ischemic cell death in brain neurons. Physiol Rev. (1999) 79:1431568. doi: 10.1152/physrev.1999.79.4.1431

22. Ayoub IM, Radhakrishnan J, Gazmuri RJ. Targeting mitochondria for resuscitation from cardiac arrest. Crit Care Med. (2008) 36:S440-6. doi: 10.1097/CCM.0b013e31818a89f4

23. Wu L, Sun HL, Gao Y, Hui KL, Xu MM, Zhong H, et al. Therapeutic hypothermia enhances cold-inducible RNA-binding protein expression and inhibits mitochondrial apoptosis in a rat model of cardiac arrest. $\mathrm{Mol}$ Neurobiol. (2017) 54:2697-705. doi: 10.1007/s12035-016-9813-6

24. Zhao N, Zhuo X, Lu Y, Dong Y, Ahmed ME, Tucker D, et al. Intranasal delivery of a caspase-1 inhibitor in the treatment of global cerebral ischemia. $\mathrm{Mol}$ Neurobiol. (2017) 54:4936-52. doi: 10.1007/s12035-016-0034-9

25. Xu X, Chua CC, Zhang M, Geng D, Liu CF, Hamdy RC, et al. The role of PARP activation in glutamate-induced necroptosis in HT-22 cells. Brain Res. (2010) 1343:206-12. doi: 10.1016/j.brainres.2010.04.080

26. Bolitho P, Voskoboinik I, Trapani J, Smyth M. Apoptosis induced by the lymphocyte effector molecule perforin. Curr Opin Immunol. (2007) 19:33947. doi: 10.1016/j.coi.2007.04.007

27. Yilmaz G, Arumugam TV, Stokes KY, Granger DN. Role of T lymphocytes and interferon-gamma in ischemic stroke. Circulation. (2006) 113:2105-12. doi: 10.1161/CIRCULATIONAHA.105.593046

28. Martin SJ, Amarante-Mendes GP, Shi L, Chuang TH, Casiano CA, O’Brien GA, et al. The cytotoxic cell protease granzyme B initiates apoptosis in a cell-free system by proteolytic processing and activation of the ICE/CED-3 family protease, CPP32, via a novel two-step mechanism. EMBO J. (1996) 15:2407-16. doi: 10.1002/j.1460-2075.1996.tb00598.x

29. Sutton VR, Davis JE, Cancilla M, Johnstone RW, Ruefli AA, Sedelies K, et al. Initiation of apoptosis by granzyme B requires direct cleavage of bid, but not direct granzyme B-mediated caspase activation. J Exp Med. (2000) 192:1403-13. doi: 10.1084/jem.192.10.1403

30. Sutton VR, Wowk ME, Cancilla M, Trapani JA. Caspase activation by granzyme B Is indirect, and caspase autoprocessing requires the release of proapoptotic mitochondrial factors. Immunity. (2003) 18:319-29. doi: 10.1016/S1074-7613(03)00050-5

Conflict of Interest: The authors declare that the research was conducted in the absence of any commercial or financial relationships that could be construed as a potential conflict of interest.

Copyright (C) $2019 \mathrm{Ji}, \mathrm{Wu}$, Shao, Meng, Xu, Zhu and Zhang. This is an open-access article distributed under the terms of the Creative Commons Attribution License (CC $B Y)$. The use, distribution or reproduction in other forums is permitted, provided the original author(s) and the copyright owner(s) are credited and that the original publication in this journal is cited, in accordance with accepted academic practice. No use, distribution or reproduction is permitted which does not comply with these terms. 